\title{
The Hedgehog Signaling Pathway is Expressed in the Adult Mouse Hypothalamus and Modulated by Fasting
}

\author{
Patrick J. Antonellis, ${ }^{1}$ Staci E. Engle, ${ }^{1}$ Kathryn M. Brewer, ${ }^{1}$ and Nicolas F. Berbari ${ }^{1,2,3}$
}

https://doi.org/10.1523/ENEURO.0276-21.2021

${ }^{1}$ Department of Biology, Indiana University-Purdue University Indianapolis, Indianapolis, Indiana 46202, ${ }^{2}$ Stark Neurosciences Research Institute, Indiana University School of Medicine, Indianapolis, Indiana 46202-3082, and ${ }^{3}$ Center for Diabetes and Metabolic Diseases, Indiana University School of Medicine, Indianapolis, Indiana 46202-3082

\begin{abstract}
The hedgehog signaling pathway is best known for its role in developmental patterning of the neural tube and limb bud. More recently, hedgehog signaling has been recognized for its roles in growth of adult tissues and maintenance of progenitor cell niches. However, the role of hedgehog signaling in fully differentiated cells like neurons in the adult brain is less clear. In mammals, coordination of hedgehog pathway activity relies on primary cilia and patients with ciliopathies such as Bardet-Biedl and Alström syndrome exhibit clinical features clearly attributable to errant hedgehog such as polydactyly. However, these ciliopathies also present with features not clearly associated with hedgehog signaling such as hyperphagia-associated obesity. How hedgehog signaling may contribute to feeding behavior is complex and unclear, but cilia are critical for proper energy homeostasis. Here, we provide a detailed analysis of the expression of core components of the hedgehog signaling pathway in the adult mouse hypothalamus with an emphasis on feeding centers. We show that hedgehog pathway genes continue to be expressed in differentiated neurons important for the regulation of feeding behavior. Furthermore, we demonstrate for the first time that pathway activity is regulated at the transcriptional level by fasting. These data suggest that hedgehog signaling is involved in the proper functioning of brain regions that regulate feeding behavior and that hedgehog pathway dysfunction may play a role in the obesity observed in certain ciliopathies.
\end{abstract}

Key words: feeding behavior; hedgehog signaling; hypothalamus; primary cilia

\section{Significance Statement}

Here we investigate the expression of hedgehog pathway components in the adult mouse hypothalamus. Using dual-labeling in situ hybridization, we show that core components of the signaling pathway are expressed in multiple neuronal cell types within the hypothalamic feeding centers. Our findings also support previous findings that astrocytes are responsive to hedgehog signaling, as determined by Gli1 and Ptch1 expression. Using quantitative PCR analysis, we show that hypothalamic hedgehog pathway activity is upregulated in response to fasting and that this response is nuclei specific. These data not only provide a more detailed understanding of hedgehog pathway expression in the adult mouse hypothalamus but also provide direct evidence of a novel role for hedgehog signaling in the physiological response to fasting.

Received June 18, 2021; accepted August 10, 2021; First published September 16, 2021.

P.J.A. is an employee of Eli Lilly Co. The authors declare no other competing financial interests.
Author contributions: P.J.A. and N.F.B. designed research; P.J.A., S.E.E., K.M.B., and N.F.B. performed research; P.J.A., S.E.E., K.M.B., and N.F.B. analyzed data; P.J.A., S.E.E., and N.F.B. wrote the paper. 


\section{Introduction}

Initially described in a Drosophila mutant screen, the hedgehog pathway is now classically recognized for its iterative role in the formation and function of several mammalian tissues and organs such as the limb bud, neural tube, and skeleton (Nüsslein-Volhard and Wieschaus, 1980; Goetz and Anderson, 2010). In mammals, the hedgehog pathway is dependent on the primary cilium as an organizing center (Huangfu et al., 2003; Breunig et al., 2008; Willaredt et al., 2008; Gorivodsky et al., 2009; Stottmann et al., 2009). Components of the hedgehog pathway such as patched (PTCH1), smoothened (SMO), and the Gli transcription factors GLI2 and GLI3 dynamically localize to the primary cilia (Corbit et al., 2005; Haycraft et al., 2005; Rohatgi et al., 2007). Upon binding to the ligand sonic hedgehog (SHH), PTCH1 leaves the cilia, allowing for SMO to enter primary cilia. GLI2 is activated into its transcriptional activator (GLI2A) form, while GLI3R formation is inhibited, leading to the expression of Gli target genes, which includes Ptch1 and Gli1 (Chen and Struhl, 1996; Marigo and Tabin, 1996; Bai et al., 2004; Fuccillo et al., 2006). Numerous other genes are known to regulate hedgehog signaling in embryonic development, such as Gpr161, an orphan G-protein-coupled receptor (GPCR) shown to localize to cilia and negatively regulate pathway activity (Mukhopadhyay et al., 2013).

Beyond embryonic development, cilia and hedgehog signaling continue to play an important role in the postnatal brain for growth and the maintenance of neural progenitors (Machold et al., 2003; Chizhikov et al., 2007; Breunig et al., 2008; Han et al., 2008; Vaillant and Monard, 2009). Primary cilia are found on almost all mammalian cell types, and their dysfunction underlies a class of human genetic disorders known as ciliopathies (Reiter and Leroux, 2017). Ciliopathies present a wide range of clinical symptoms, some of which are associated with genetic defects in hedgehog signaling such as bone, limb patterning, and genitalia malformations (Umehara et al., 2000; Gao et al., 2001; Hellemans et al., 2003; Mujahid et al., 2018; Khan et al., 2019). Furthermore, the ciliopathy Carpenter syndrome [\#20100, Online Mendelian Inheritance in Man (OMIM)] results from mutations in Rab23, a negative regulator of hedgehog signaling. Interestingly, obesity is also a core clinical phenotype of certain ciliopathies such as Bardet-Biedl syndrome (\#209900, OMIM), Alström syndrome (\#203800, OMIM), and Carpenter syndrome (Jenkins et al., 2007; Alessandri et al., 2010; Marshall et al., 2011; Forsythe et al., 2018). Thus, these genetic disorders implicate a potential role for

This work was funded by National Institute of Diabetes and Digestive and Kidney Diseases Grant R01-DK-114008 to N.F.B.

Correspondence should be addressed to Nicolas F. Berbari at nberbari@ iupui.edu.

https://doi.org/10.1523/ENEURO.0276-21.2021

Copyright (C) 2021 Antonellis et al.

This is an open-access article distributed under the terms of the Creative Commons Attribution 4.0 International license, which permits unrestricted use, distribution and reproduction in any medium provided that the original work is properly attributed. hedgehog signaling in regulation of energy homeostasis in humans.

Ciliopathy mouse models, as well as conditional animal models of cilia loss, have implicated hypothalamic neuronal cilia-mediated feeding behaviors in obesity (Davenport et al., 2007; Siljee et al., 2018; Wang et al., 2019; Rouabhi et al., 2021; Sun et al., 2021). Neuronal primary cilia preferentially localize GPCRs known to be important for the regulation of energy homeostasis and feeding behavior, such as neuropeptide $Y$ (NPY) receptors 2 and 5, melanin-concentrating hormone receptor 1 (MCHR1) and melanocortin-4 receptor (MC4R; Berbari et al., 2008; Loktev and Jackson, 2013; Siljee et al., 2018). However, the impact of ciliary localization on their signaling capabilities is not well understood. We have previously demonstrated in primary mouse hypothalamic neurons in vitro, interactions between a ciliary GPCR and hedgehog signaling, suggesting that the hedgehog pathway may modulate GPCR activity at the cilium in differentiated neurons (Bansal et al., 2019). However, the role of hedgehog signaling in vivo in the adult hypothalamus is less clear. In situ hybridization studies have shown that Shh, Ptch1, and Smo mRNA are expressed in several regions of adult rat brain, including the hypothalamus (Traiffort et al., 1998, 1999, 2001; Banerjee et al., 2005). Here we characterize in greater detail the expression and transcriptional activity of the hedgehog pathway in the feeding centers of the adult hypothalamus in vivo. We also demonstrate that hedgehog pathway activity changes based on feeding status and that this response is absent following the onset of obesity, suggesting a role for hedgehog signaling in the modulation of adult behaviors.

\section{Materials and Methods}

\section{Animals}

All procedures were approved by the Institutional Animal Care and Use Committee at Indiana UniversityPurdue University Indianapolis. Male C57BL/6J mice (stock \#000664) were ordered from The Jackson Laboratory, and they were housed on a standard $12 \mathrm{~h}$ light/dark cycle and given food and water ad libitum except for experiments as described previously. Chow-fed mice were maintained on a standard chow diet consisting of $13 \%$ fat, $67 \%$ carbohydrate, and $20 \%$ protein caloric content (2014 Teklad, Envigo). High-fat diet (HFD)-fed animals were maintained on a diet consisting of $40 \%$ fat, $39 \%$ carbohydrate, and $21 \%$ protein caloric content starting at 6 weeks of age (catalog \#TD95217, Envigo).

\section{In situ hybridization}

Brains from 8- to 10-week-old male C57BL/6J mice were harvested and fixed as described previously (Engle et al., 2018). Sections were cut at a thickness of $15 \mu \mathrm{m}$ and mounted directly on slides, then were postfixed with $4 \%$ paraformaldehyde for $16 \mathrm{~h}$ at $4^{\circ} \mathrm{C}$. The detection of transcripts in brain sections was performed using the RNAscope 2.5 HD Duplex Assay (ACD). Tissue pretreatment was performed according to user manual technical note 320534 Rev A. Probe hybridization, counterstaining, 

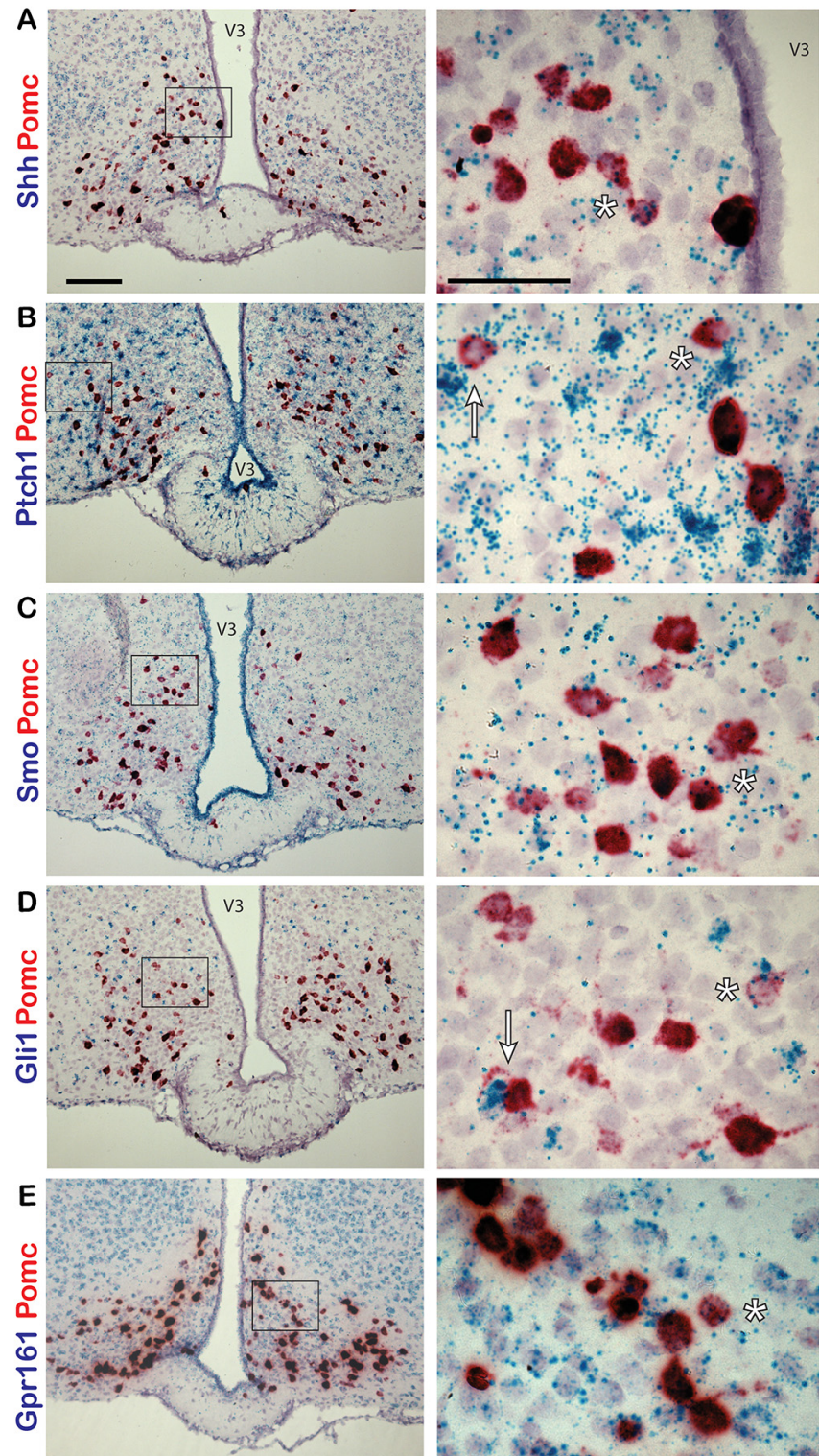
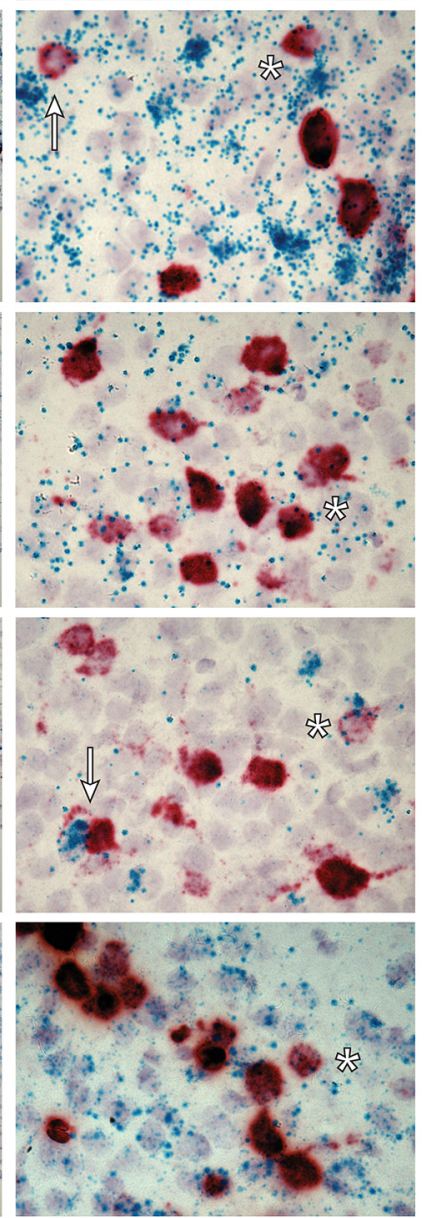
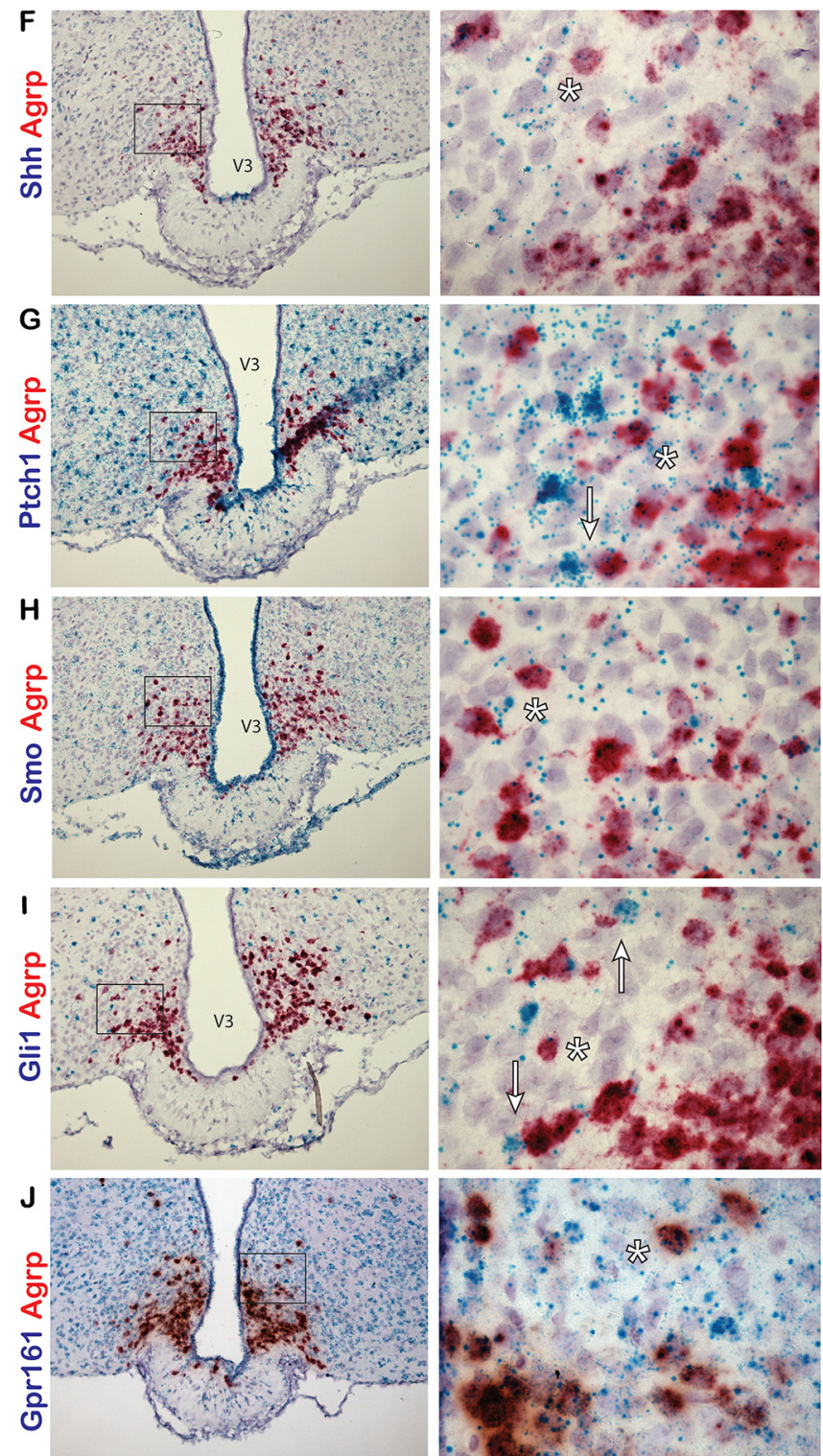
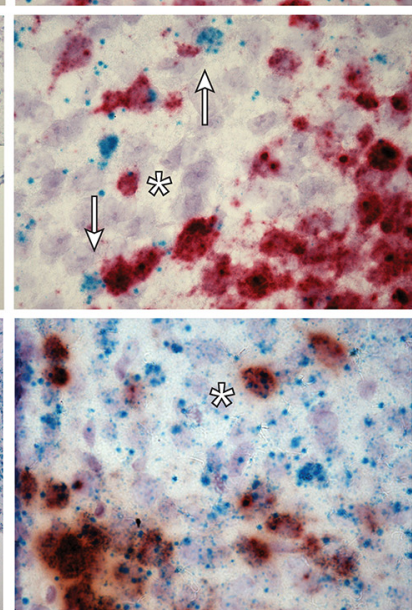

Figure 1. Hedgehog pathway expression in the adult mouse ARC. Dual-probe in situ hybridization labeling of the ARC with probes to hedgehog pathway and neuronal gene transcripts. $\boldsymbol{A}-\boldsymbol{J}$, Pomc $(\boldsymbol{A}-\boldsymbol{E})$ and Agrp $(\boldsymbol{F}-\boldsymbol{J})$ probes are labeled in red, while Shh $(\boldsymbol{A}, \boldsymbol{F})$, Ptch1 $(\boldsymbol{B}, \boldsymbol{G})$, Smo $(\boldsymbol{C}, \boldsymbol{H})$, Gli1 $(\boldsymbol{D}, \boldsymbol{I})$, and $\operatorname{Gpr161}(\boldsymbol{E}, \boldsymbol{J})$ probes are labeled in blue. Examples of cells colabeled by both probes are denoted by an asterisk (*). Pomc- or Agrp-expressing cells adjacent to highly expressing Ptch1 (B, G) or Gli1 (D, I) cells are denoted by an arrow. Right-hand panels are magnified images of the region shown in a black box on the left-hand side. Scale bars: left panels, $100 \mu \mathrm{m}$; right panels, $50 \mu \mathrm{m}$. V3, Third ventricle.

and mounting of slides were performed according to user manual \#322500-USM Rev A. Slides were assayed using probes to SHH (catalog \#314361), SMO (catalog \#318411), GLI1 (catalog \#311001), PTCH1 (catalog \#402811), GPR161 (G-protein-coupled receptor 161; catalog \#318111), AGRP (agouti-related peptide; catalog \#400711-C2), POMC (proopiomelanocortin; catalog \#314081-C2), MC4R (catalog \#319181-C2), MCHR1 (catalog \#317491-C2), or GFAP (glial fibrillary acidic protein; catalog \#313211-C2) transcripts (ACD). Sections were counterstained with hematoxylin, dehydrated, and mounted using VectaMount (Vector Laboratories). Slides with positive control probe (PPIB-C1/POLR2A-C2; ACD catalog \#321651) and negative control probe (DapB; ACD catalog \#320751) were run with each experiment. At least three animals were analyzed for each group.

\section{Quantitative real-time PCR}

RNA was isolated, cDNA was prepared, and quantitative real-time PCR was performed as described previously (Bansal et al., 2019). Assays-on-Demand Gene Expression Probes (Applied Biosystems) were as follows: Shh Mm00436528_m1; Ptch1 Mm00436026_m1; Smo Mm01162710_m1; Gli1 Mm00494654_m1; and Gpr161: Mm01291057_m1. Ct values were normalized to $\beta$-actin, relative expression was calculated by the 

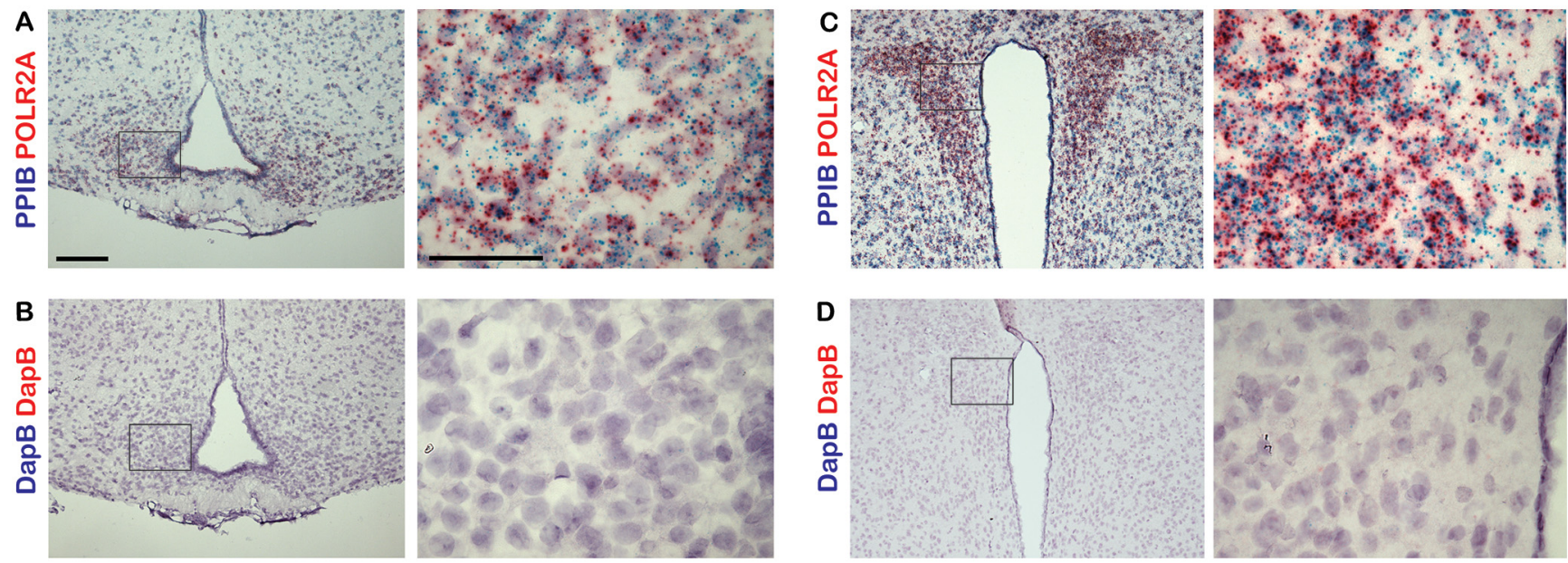

Figure 2. Controls for dual-probe in situ hybridization studies. $\boldsymbol{A}-\boldsymbol{D}$, Sections of adult mouse ARC and PVN were labeled with either positive control probes targeting the mouse genes Ppib and Polr2a $(\boldsymbol{A}, \boldsymbol{C})$ or negative control probes targeting the bacterial gene $\operatorname{dap} B(\boldsymbol{B}, \boldsymbol{D})$. Right-hand panels are magnified images of a region shown in a black box on the left-hand side. Scale bars: left panels, $100 \mu \mathrm{m}$; right panels, $50 \mu \mathrm{m}$.

$\Delta \Delta \mathrm{Ct}$ method, and fold change was calculated by normalizing relative expression to the proper control.

\section{Experimental design and statistical analyses}

Whole hypothalamus was collected from 35- to 36week-old C57BL/6 lean and obese animals that were allowed either ad libitum access to food or were fasted overnight. Region-specific micropunches were collected from 7- to 8-week-old, lean, C57BL/6 mice using $1.0 \mathrm{~mm}$ Militex Biopsy Punch (Electron Microscopy Sciences). There was a minimum of six animals per treatment group. Statistical analysis was performed using two-way ANOVA and corrected for multiple comparisons. Differences were considered significant when $p<0.05$. Data are presented as the mean \pm SEM.

\section{Results}

To determine whether Shh and components of the signaling pathway are expressed in the adult mouse hypothalamus, we performed in situ hybridization studies. Using a dual-labeling approach, we first assessed whether neurons of one of the feeding centers, the arcuate nucleus of the hypothalamus (ARC), express hedgehog pathway genes. Two major neuronal subtypes within the ARC, the anorexigenic POMC-expressing neurons and orexigenic AGRP/NPY-coexpressing neurons, are crucial for normal energy homeostasis (Belgardt et al., 2009). Hypothalamic sections from adult, C57BL/6 mice were labeled with probes to Shh, Ptch1, Smo, Gli1, or Gpr161 and colabeled with probes to either Pomc or Agrp. In the ARC, we found that all hedgehog pathway genes assayed were coexpressed in neurons expressing Pomc (Fig. 1A-D) and Agrp (Fig. 1F-l). We also found that Gpr161 is expressed at a relatively high level throughout the ARC (Fig. 1E,J). For each experiment, sections were labeled with positive and negative control probes (Fig. 2).

We next assessed whether Shh and its pathway components are also expressed in another feeding center, the paraventricular nucleus of the hypothalamus (PVN). We labeled sections of hypothalamus with probes to Shh, Ptch1, Smo, Gli1, or Gpr161, and colabeled them with probes to either Mchr1 or Mc4r. Both MCHR1 and MC4R are known to be important for the regulation of energy homeostasis and feeding behavior and are localized to primary cilia in neurons (Berbari et al., 2008; Siljee et al., 2018). In the PVN, we observed relatively few Mc4r-positive neurons with sparse incidence of colabeling with probes to hedgehog pathway genes (Fig. $3 A-D)$. However, Mchr1-positive neurons were much more abundant and were frequently colabeled with probes to all hedgehog pathway genes used (Fig. 3FI). As in the ARC, Gpr161 is expressed abundantly throughout the PVN (Fig. 3E,J).

Interestingly, we observed cells highly positive for either Gli1 or Ptch1 expression throughout the ARC and PVN. Some of these cells appeared adjacent to neurons expressing Pomc, Agrp, or Mchr1 (Figs. 1B,D,G,I, 3l, arrows). Previously, it has been reported that astrocytes of the adult mouse brain are responsive to hedgehog signaling and increase Gli1 expression on pathway activation (Garcia et al., 2010). Therefore, we sought to determine whether these hedgehog-responsive cells were astrocytes by colabeling with probes to either Gli1 or Ptch1, and Gfap, an astrocyte marker. In the ARC (Fig. 4A,C) and PVN (Fig. 4B,D), cells highly expressing Gfap were observed colabeled with Gli1 or Ptch1, suggesting that some of the hedgehog-responsive cells adjacent to neurons may be astrocytes in the hypothalamus.

Since we determined that genes of the hedgehog pathway are expressed in neurons of the adult hypothalamus, we next wanted to determine whether these genes are transcriptionally regulated by physiological changes associated with the normal function of this brain region, such as nutritional status. This was accomplished by gene expression analysis of the whole hypothalamus from chow-fed lean and HFD-fed obese animals in the fed and fasted state. HFD-fed animals weighed significantly more 

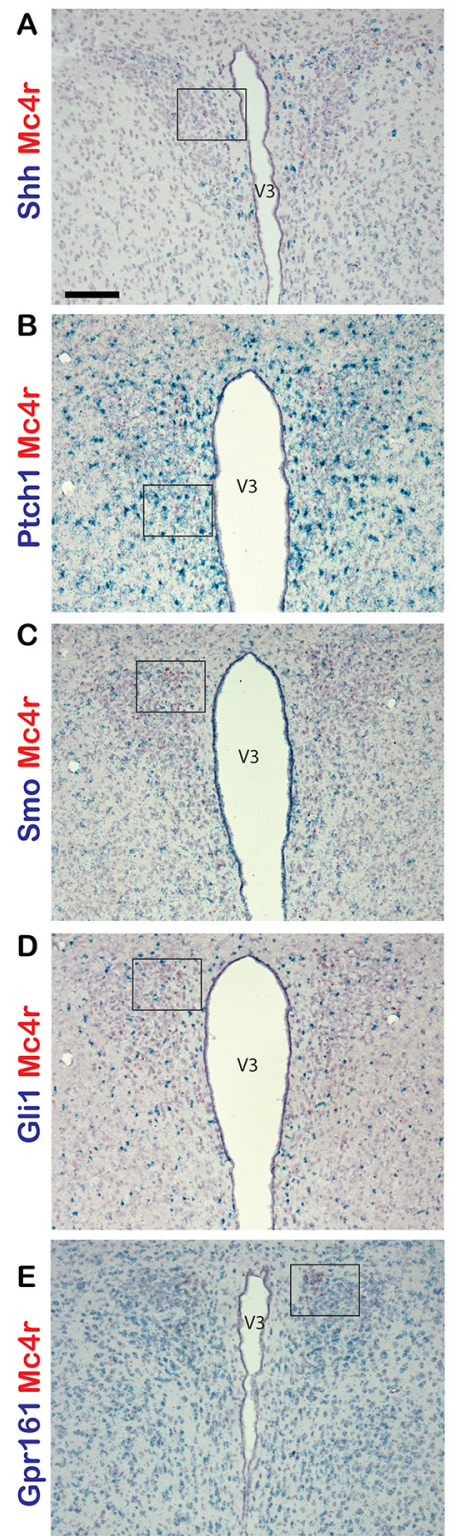
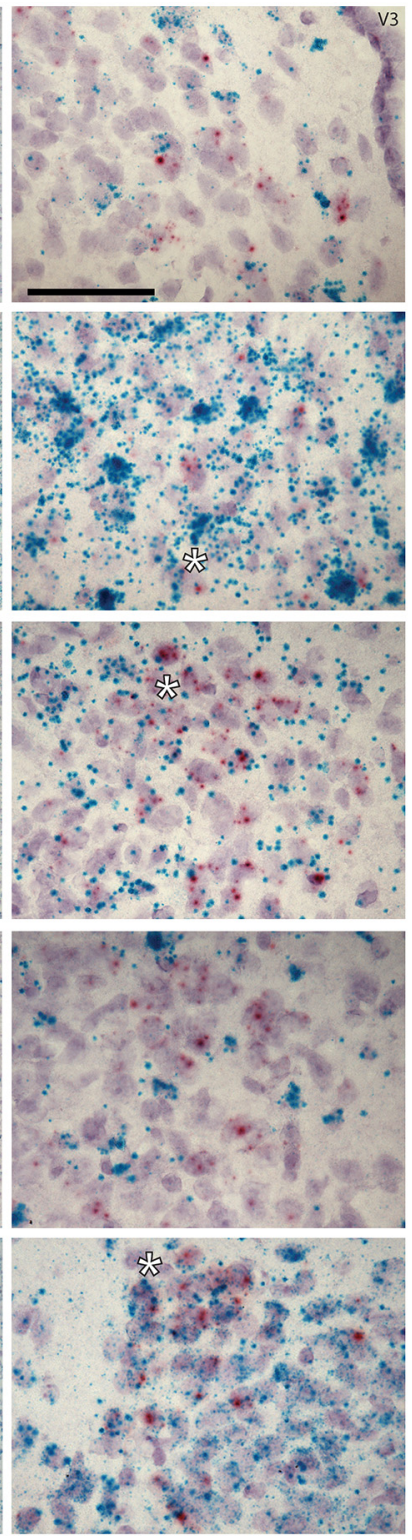
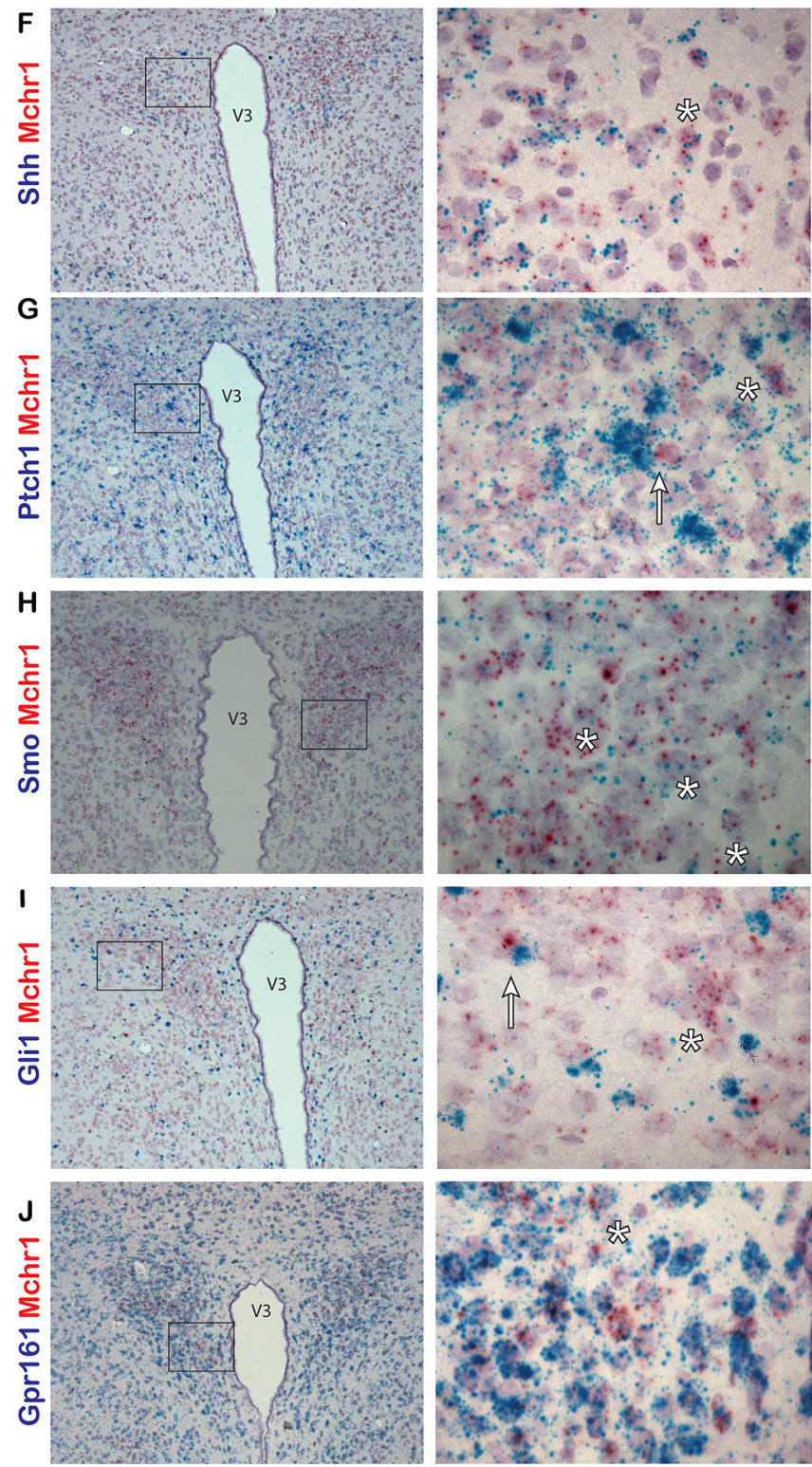

Figure 3. Hedgehog pathway expression in adult mouse PVN. A-J, Dual-probe in situ hybridization of probes for hedgehog pathway gene transcripts colabeled with probes to neuronal gene transcripts in the PVN of adult mice. $M c 4 r(\boldsymbol{A}-\boldsymbol{E})$ and $M c h r 1(\boldsymbol{F}-\boldsymbol{J})$ probes are labeled in red, while Shh $(\boldsymbol{A}, \boldsymbol{F})$, Ptch1 $(\boldsymbol{B}, \boldsymbol{G})$, Smo $(\boldsymbol{C}, \boldsymbol{H})$, Gli1 $(\boldsymbol{D}, \boldsymbol{I})$, and Gpr161 (E, $\boldsymbol{J})$ probes are labeled in blue. Example of cells colabeled by both probes are denoted by an asterisk $\left(^{*}\right)$. Mchr1-expressing cells adjacent to highly expressing Gli1 cells are denoted by an arrow (I). Right-hand panels are magnified images of a region in a black box on the left hand-side. Scale bars: left panels, $100 \mu \mathrm{m}$; right panels, $50 \mu \mathrm{m}$. V3, Third ventricle.

than their chow-fed counterparts at 35 weeks of age $(57.1 \pm 0.97$ vs $34.8 \pm 0.75 \mathrm{~g} ;$ mean $\pm \mathrm{SEM} ; p<0.05$ Student's $t$ test). Lean and obese animals were allowed either ad libitum access to food or were fasted overnight, then whole hypothalamic RNA was collected for quantitative PCR (qRT-PCR) analysis. In lean animals, there was a significant increase in both Shh and Gli1 expression in the hypothalamus after an overnight fast (Fig. 5A). The expression of Ptch1 also was elevated in fasted compared with fed animals, but this effect was not significant (Fig. $5 A)$. Strikingly, there were no significant changes in gene expression in the hypothalamus of obese fasted versus fed animals (Fig. 5A). Since transcriptional regulation was observed in response to fasting in control chow-fed animals at the level of the whole hypothalamus, we wanted to assess which specific nuclei were contributing to this effect. Once again, control, normal weight C57BL/6J animals were allowed either ad libitum access to food or were fasted overnight. Micropunches were then collected from the cortex, ventromedial hypothalamus (VMH), PVN, and $A R C$ for $\mathrm{qPCR}$ analysis. We found that fasting that induces an increase in Gli1 expression in the whole hypothalamus is driven by increases in Gli1 expression specifically in the VMH and PVN but not in the ARC (Fig. 5B). We 

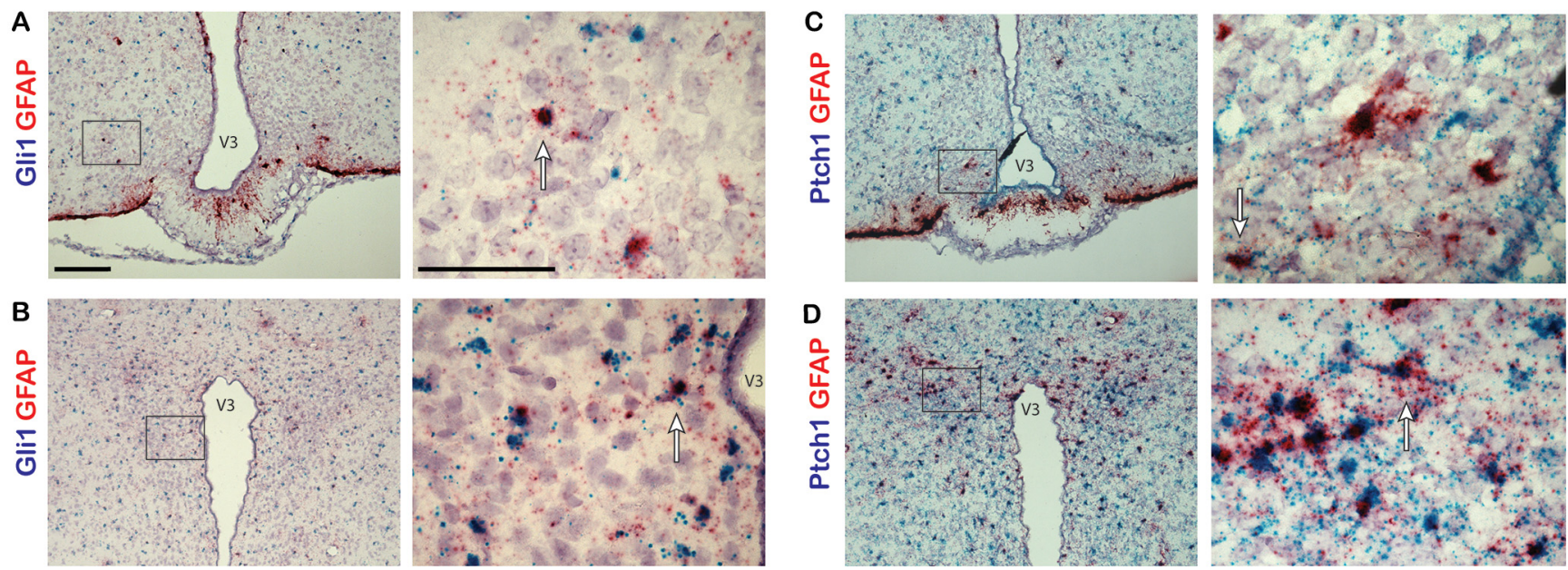

Figure 4. Gli1 and Ptch1 expression in astrocytes in adult mouse hypothalamus. Dual-probe in situ hybridization was performed with probes for Gli1 or Ptch1 and astrocyte marker Gfap gene transcripts. $\boldsymbol{A}-\boldsymbol{D}$, Sections of the ARC $(\boldsymbol{A}, \boldsymbol{C})$ and PVN $(\boldsymbol{B}, \boldsymbol{D})$ from adult mice were colabeled with probes to Gli1 or Ptch1 labeled in blue and probes to Gfap labeled in red. Example cells that are highly positive for both probes are denoted by an arrow. Right-hand panels are magnified images of a region shown in a black box on the left-hand side. Scale bars: left panels, $100 \mu \mathrm{m}$; right panels, $50 \mu \mathrm{m}$. V3, Third ventricle.

also found that Gli1 was upregulated, to a lesser extent, in the cortex (Fig. 5B). Additionally, expression of Smo was increased in the $\mathrm{VMH}$, while Shh gene expression was reduced in the ARC following an overnight fast (Fig. 5B). Finally, we performed qPCR on whole hypothalamus and cortex, a brain region known to exhibit hedgehog pathway activity (Garcia et al., 2010; Harwell et al., 2012; Hill et al., 2019), collected from adult animals. We found that all hedgehog pathway genes measured were more highly expressed in the hypothalamus than the cortex (Fig. 6). Overall, these data demonstrate that not only does expression of the hedgehog pathway continue into adulthood in the hypothalamus, a region critical for energy homeostasis, but that specific nuclei respond with transcriptional changes based on feeding status.

\section{Discussion}

Primary cilia are crucial for mediating hedgehog signaling in mammals and, furthermore, certain ciliopathies are associated with pediatric obesity (Engle et al., 2021). Therefore, we focused our efforts on evaluating the expression of the hedgehog pathway in hypothalamic feeding centers of adult mice. Because reliable commercial antibodies for many pathway proteins are currently unavailable, making immunolabelling studies difficult, we used a dual-labeling in situ hybridization approach that allowed for sensitive detection of hedgehog pathway gene transcripts in specific adult neurons. Our in situ data revealed the broad expression of Shh and core pathway components throughout the hypothalamus of adult mice. We found that mRNA for Shh, Ptch1, Smo, Gli1, and Gpr161 could be detected in both the ARC and PVN. Within the ARC, each one of these transcripts are detected in neurons coexpressing Pomc or Agrp. While a similar labeling pattern was observed in the PVN, transcripts for hedgehog pathway genes were more readily found colabeled with probes to Mchr1 than Mc4r.
However, this could potentially be because of the relatively low abundance of MC4R-expressing neurons or low mRNA expression for this particular GPCR. Our results expand on prior studies that found hedgehog pathway expression in the adult rat brain (Traiffort et al., 1998, 1999; Banerjee et al., 2005) by demonstrating that neurons in two nuclei of the hypothalamus important for regulation of feeding behavior express Shh and members of its signaling pathway. Somewhat surprisingly, we also found that Gpr161 is expressed abundantly throughout both the ARC and PVN. This is contrary to previously published findings that used digoxigenin probes for in situ analysis of the adult mouse brain and showed a more restricted expression pattern of Gpr161 in the nucleus accumbens and amygdala with low hypothalamic expression (Ehrlich et al., 2018). Analysis of the amygdala and accumbens was outside the scope of the present study, and relative expression of Gpr161 between these brain regions was not determined. However, a thorough understanding of this GPCR-negative regulator of hedgehog signaling in the adult brain may reveal themes for its roles in ciliamediated behaviors.

Interestingly, in both the ARC and PVN we observed cells with high expression of either Ptch1 or Gli1 immediately adjacent to neurons expressing Pomc, Agrp, or Mchr1. By colabeling sections of hypothalamus with probes to Gfap and Gli1 or Ptch1, we were able to identify some of these hedgehog-responsive cells as Gfap-positive astrocytes. While neurons of the adult mouse hypothalamus express Shh and components required for its signal transduction, the cells that are most responsive to hedgehog signaling, as indicated by high levels of Gli1 and Ptch 1 expression, may in fact be astrocytes. This supports previous findings showing that not only do neurons produce Shh, but subpopulations of mature astrocytes in the forebrain are responsive to hedgehog signaling (Garcia et al., 2010). It remains unclear why 
A

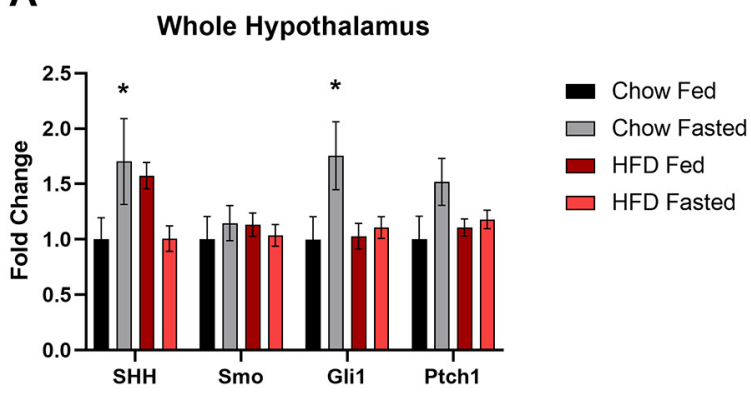

B
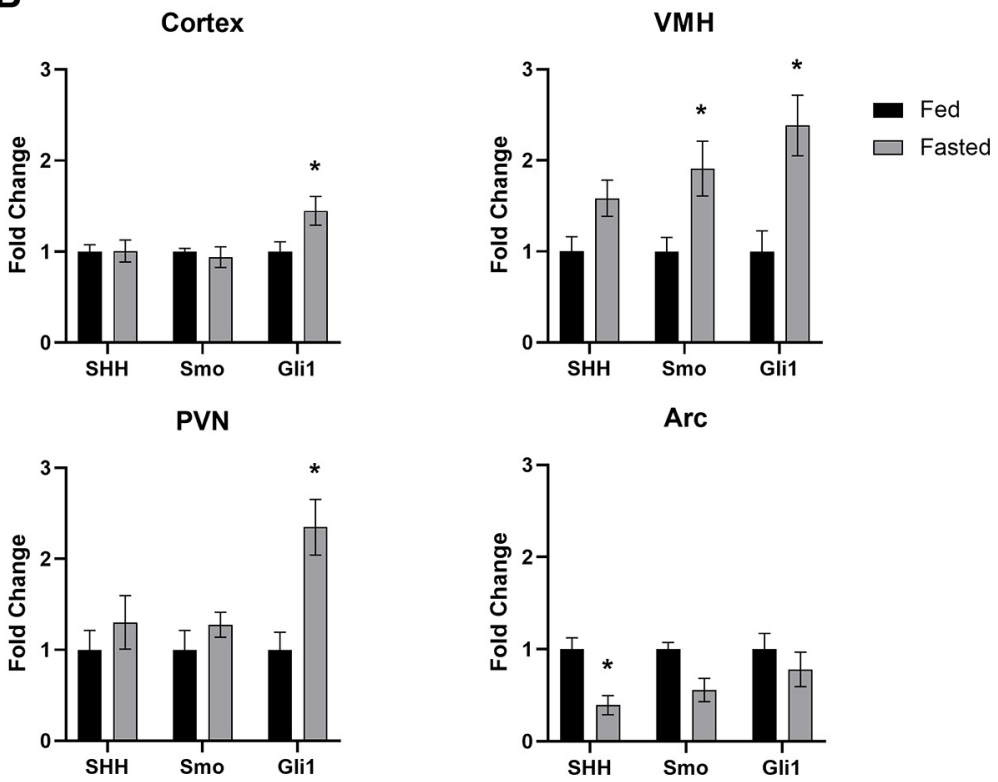

Figure 5. Feeding status mediated changes in hypothalamic hedgehog pathway gene expression. A, Hedgehog pathway gene expression in the whole hypothalamus of adult mice. Lean animals fed a standard chow diet or obese animals fed a high-fat diet were allowed ad libitum access to food or fasted overnight. A minimum of six animals were used per treatment group. Whole hypothalamic RNA was used for qPCR. B, Hedgehog pathway gene expression in brain micropunches of adult mice. A total of 16 animals, 8 per treatment group, fed a chow diet were allowed ad libitum access to food or fasted overnight. Micropunches were taken from specific nuclei of the hypothalamus and cortex for qPCR analysis. ${ }^{*} p<0.05$.

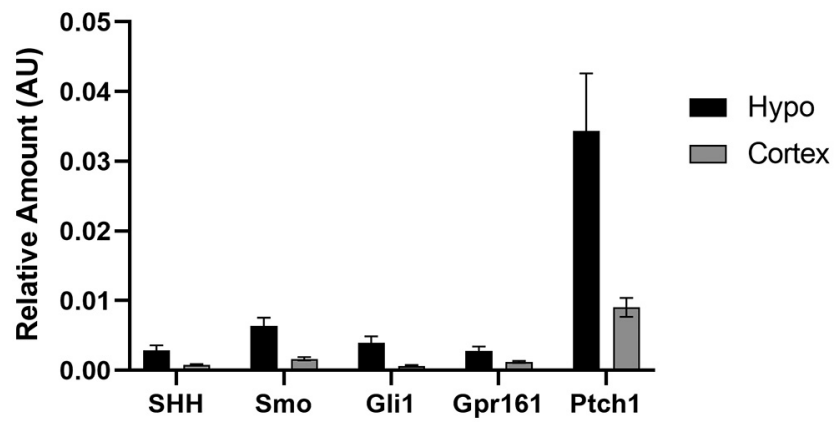

Figure 6. Expression of selected hedgehog pathway transcripts in the adult mouse brain. Whole hypothalamus and cortex were collected for qPCR analysis from six adult animals allowed ad libitum access to food. these Gfap-positive cells outside of known neurogenic niches exhibit high levels of Gli1 and Ptch1 relative to neighboring neurons.

There is growing evidence suggesting that the hedgehog pathway is involved in the regulation of whole-body energy homeostasis. It has been demonstrated in both the fat body of Drosophila and the adipose tissue of mice that hedgehog signaling regulates adipocyte differentiation (Suh et al., 2006; Pospisilik et al., 2010). Circulating forms of Hedgehog have been detected, and during Drosophila larval development have been shown to be secreted from the gut and to act on multiple tissues to coordinate development with nutrient availability (Palm et al., 2013; Rodenfels et al., 2014). Given these findings, we also analyzed the expression of Shh, Smo, and Gli1 in the adult mouse hypothalamus in response to changes in metabolic state and nutritional status. We compared transcriptional regulation of the hedgehog pathway in both lean control diet-fed animals and obese HFD-fed animals. 
Animals fed an HFD are a well established model of noninsulin-dependent type II diabetes, which exhibit many hallmarks of metabolic dysfunction such as reduced glucose tolerance (Surwit et al., 1988; Fontaine and Davis, 2016). In the whole hypothalamus, the expression of Gli1 was upregulated in lean mice following an overnight fast. Congruent with this finding, the expression of Ptch1 exhibited a nonsignificant upregulation in lean, fasted animals. In contrast, we observed no significant transcriptional regulation in the hypothalamus of fasted obese animals compared with fed controls. These data suggest that hedgehog signaling is involved in the physiological response to fasting and may be dysregulated in obese animals. To determine whether this fasting-induced upregulation of Gli1 is a response generated by the whole hypothalamus or specific nuclei, micropunches were collected from hypothalamic nuclei as well as the cortex. We found that in fasted animals Gli1 expression was elevated specifically in the VMH, PVN, and, to a lesser extent, the cortex, but not the ARC. These data suggest that increased hedgehog pathway activity in the hypothalamus upon fasting is primarily driven by the VMH and PVN. Future studies will determine the cell types responsible within these nuclei for this increase in Gli1 expression. Additional work is also required to determine the source of the pathway activation observed in these studies. It is possible that ligand is produced outside of the hypothalamus, therefore, further analysis could potentially reveal the primary source of Shh following an overnight fast.

Taken as a whole, these data show that neurons of the hypothalamus express both Shh and members of its signaling pathway that are required for signal transduction, and that activity of this pathway is upregulated in response to fasting in discrete hypothalamic nuclei. Given our in situ data identifying astrocytes as being highly positive for Gli1 and Ptch1 in the hypothalamus, it is possible that astrocytes or other support cells are primarily responsible for this fasting-induced upregulation of hedgehog signaling. We have previously shown in primary hypothalamic cultures, consisting of both neurons and glia, that modulation of the hedgehog pathway alters the electrophysiological response to melanin-concentrating hormone (Bansal et al., 2019). Interestingly, it has also been demonstrated in primary cortical cultures that the presence of astrocytes alters the response of neurons to agonism of the hedgehog pathway (Ugbode et al., 2017). Furthermore, astrocyte-specific inhibition of hedgehog signaling in vivo was shown to disrupt early postnatal organization and remodeling of cortical synapses resulting in increased neuronal excitability (Hill et al., 2019). Together, these findings suggest novel potential roles for hedgehog signaling outside of its roles as a classical developmental morphogen or in stem cell niche regulation.

The data presented here on the expression and transcriptional regulation of the hedgehog pathway in the adult mouse hypothalamus lays the foundation for future mechanistic studies to determine its role in the proper functioning of the hypothalamus. Given that mammalian hedgehog signaling is coordinated by primary cilia, our future studies will focus on how hypothalamic hedgehog expression may contribute to the obesity phenotype seen in ciliopathies such as Bardet-Biedl syndrome and Alström syndrome (Marshall et al., 2011; Forsythe et al., 2018). Interestingly, certain ciliopathy clinical features such as skeletal and external genitalia abnormalities are also observed in patients with genetic defects in the hedgehog pathway (Umehara et al., 2000; Gao et al., 2001; Hellemans et al., 2003; Mujahid et al., 2018; Khan et al., 2019). Therefore, it would be of interest to determine whether hedgehog signaling is dysregulated in the hypothalamus of animal ciliopathy models. Further mechanistic studies are needed to determine whether hedgehog signaling modulates neuronal activity critical for the physiological response to fasting and whether genetic modulation of the hedgehog pathway in the hypothalamus alters feeding behavior. In conclusion, elucidating the involvement of this developmentally important signaling pathway in feeding behavior and body composition is an exciting new avenue of the hedgehog pathway to explore. Greater understanding of the hedgehog pathway in adult energy homeostasis may also reveal common themes for this pathway in regulation of other behaviors.

\section{References}

Alessandri JL, Dagoneau N, Laville JM, Baruteau J, Hébert JC, Cormier-Daire V (2010) RAB23 mutation in a large family from Comoros Islands with Carpenter syndrome. Am J Med Genet A 152A:982-986.

Bai CB, Stephen D, Joyner AL (2004) All mouse ventral spinal cord patterning by hedgehog is Gli dependent and involves an activator function of Gli3. Dev Cell 6:103-115.

Banerjee SB, Rajendran R, Dias BG, Ladiwala U, Tole S, Vaidya VA (2005) Recruitment of the Sonic hedgehog signalling cascade in electroconvulsive seizure-mediated regulation of adult rat hippocampal neurogenesis. Eur J Neurosci 22:1570-1580.

Bansal R, Engle SE, Antonellis PJ, Whitehouse LS, Baucum AJ, 2nd, Cummins TR, Reiter JF, Berbari NF (2019) Hedgehog Pathway Activation Alters Ciliary Signaling in Primary Hypothalamic Cultures. Front Cell Neurosci 13:266.

Belgardt BF, Okamura T, Brüning JC (2009) Hormone and glucose signalling in POMC and AgRP neurons. J Physiol 587:5305-5314.

Berbari NF, Johnson AD, Lewis JS, Askwith CC, Mykytyn K (2008) Identification of ciliary localization sequences within the third intracellular loop of $\mathrm{G}$ protein-coupled receptors. Mol Biol Cell 19:1540-1547.

Breunig JJ, Sarkisian MR, Arellano JI, Morozov YM, Ayoub AE, Sojitra S, Wang B, Flavell RA, Rakic P, Town T (2008) Primary cilia regulate hippocampal neurogenesis by mediating sonic hedgehog signaling. Proc Natl Acad Sci U S A 105:13127-13132.

Chen Y, Struhl G (1996) Dual roles for patched in sequestering and transducing Hedgehog. Cell 87:553-563.

Chizhikov VV, Davenport J, Zhang Q, Shih EK, Cabello OA, Fuchs JL, Yoder BK, Millen KJ (2007) Cilia proteins control cerebellar morphogenesis by promoting expansion of the granule progenitor pool. J Neurosci 27:9780-9789.

Corbit KC, Aanstad P, Singla V, Norman AR, Stainier DY, Reiter JF (2005) Vertebrate Smoothened functions at the primary cilium. Nature 437:1018-1021.

Davenport JR, Watts AJ, Roper VC, Croyle MJ, van Groen T, Wyss JM, Nagy TR, Kesterson RA, Yoder BK (2007) Disruption of intraflagellar transport in adult mice leads to obesity and slow-onset cystic kidney disease. Curr Biol 17:1586-1594.

Ehrlich AT, Maroteaux G, Robe A, Venteo L, Nasseef MT, van Kempen LC, Mechawar N, Turecki G, Darcq E, Kieffer BL (2018) Expression map of 78 brain-expressed mouse orphan GPCRs 
provides a translational resource for neuropsychiatric research. Commun Biol 1:102.

Engle SE, Antonellis PJ, Whitehouse LS, Bansal R, Emond MR, Jontes JD, Kesterson RA, Mykytyn K, Berbari NF (2018) A CreER mouse to study melanin concentrating hormone signaling in the developing brain. Genesis 56:e23217.

Engle SE, Bansal R, Antonellis PJ, Berbari NF (2021) Cilia signaling and obesity. Semin Cell Dev Biol 110:43-50.

Fontaine DA, Davis DB (2016) Attention to background strain is essential for metabolic research: C57BL/6 and the International Knockout Mouse Consortium. Diabetes 65:25-33.

Forsythe E, Kenny J, Bacchelli C, Beales PL (2018) Managing Bardet-Biedl syndrome-now and in the future. Front Pediatr 6:23.

Fuccillo M, Joyner AL, Fishell G (2006) Morphogen to mitogen: the multiple roles of hedgehog signalling in vertebrate neural development. Nat Rev Neurosci 7:772-783.

Gao B, Guo J, She C, Shu A, Yang M, Tan Z, Yang X, Guo S, Feng G, $\mathrm{He} L$ (2001) Mutations in IHH, encoding Indian hedgehog, cause brachydactyly type A-1. Nat Genet 28:386-388.

Garcia AD, Petrova R, Eng L, Joyner AL (2010) Sonic hedgehog regulates discrete populations of astrocytes in the adult mouse forebrain. J Neurosci 30:13597-13608.

Goetz SC, Anderson KV (2010) The primary cilium: a signalling centre during vertebrate development. Nat Rev Genet 11:331-344.

Gorivodsky M, Mukhopadhyay M, Wilsch-Braeuninger M, Phillips M, Teufel A, Kim C, Malik N, Huttner W, Westphal H (2009) Intraflagellar transport protein 172 is essential for primary cilia formation and plays a vital role in patterning the mammalian brain. Dev Biol 325:24-32.

Han YG, Spassky N, Romaguera-Ros M, Garcia-Verdugo JM, Aguilar A, Schneider-Maunoury S, Alvarez-Buylla A (2008) Hedgehog signaling and primary cilia are required for the formation of adult neural stem cells. Nat Neurosci 11:277-284.

Harwell CC, Parker PR, Gee SM, Okada A, McConnell SK, Kreitzer AC, Kriegstein AR (2012) Sonic hedgehog expression in corticofugal projection neurons directs cortical microcircuit formation. Neuron 73:1116-1126.

Haycraft CJ, Banizs B, Aydin-Son Y, Zhang Q, Michaud EJ, Yoder BK (2005) Gli2 and Gli3 localize to cilia and require the intraflagellar transport protein polaris for processing and function. PLoS Genet 1:e53.

Hellemans J, Coucke PJ, Giedion A, De Paepe A, Kramer P, Beemer F, Mortier GR (2003) Homozygous mutations in IHH cause acrocapitofemoral dysplasia, an autosomal recessive disorder with coneshaped epiphyses in hands and hips. Am J Hum Genet 72:10401046.

Hill SA, Blaeser AS, Coley AA, Xie Y, Shepard KA, Harwell CC, Gao WJ, Garcia ADR (2019) Sonic hedgehog signaling in astrocytes mediates cell type-specific synaptic organization. Elife 8:e45545.

Huangfu D, Liu A, Rakeman AS, Murcia NS, Niswander L, Anderson KV (2003) Hedgehog signalling in the mouse requires intraflagellar transport proteins. Nature 426:83-87.

Jenkins D, Seelow D, Jehee FS, Perlyn CA, Alonso LG, Bueno DF, Donnai D, Josifova D, Josifiova D, Mathijssen IMJ, Morton JEV, Orstavik KH, Sweeney E, Wall SA, Marsh JL, Nurnberg P, PassosBueno MR, Wilkie AOM (2007) RAB23 mutations in Carpenter syndrome imply an unexpected role for hedgehog signaling in cranialsuture development and obesity. Am J Hum Genet 80:1162-1170.

Khan S, Lin S, Harlalka GV, Ullah A, Shah K, Khalid S, Mehmood S, Hassan MJ, Ahmad W, Self JE, Crosby AH, Baple EL, Gul A (2019) BBS5 and INPP5E mutations associated with ciliopathy disorders in families from Pakistan. Ann Hum Genet 83:477-482.

Loktev AV, Jackson PK (2013) Neuropeptide Y family receptors traffic via the Bardet-Biedl syndrome pathway to signal in neuronal primary cilia. Cell Rep 5:1316-1329.

Machold R, Hayashi S, Rutlin M, Muzumdar MD, Nery S, Corbin JG, Gritli-Linde A, Dellovade T, Porter JA, Rubin LL, Dudek H, McMahon AP, Fishell G (2003) Sonic hedgehog is required for progenitor cell maintenance in telencephalic stem cell niches. Neuron 39:937-950.
Marigo V, Tabin CJ (1996) Regulation of patched by sonic hedgehog in the developing neural tube. Proc Natl Acad Sci U S A 93:93469351.

Marshall JD, Maffei P, Collin GB, Naggert JK (2011) Alström syndrome: genetics and clinical overview. Curr Genomics 12:225235.

Mujahid S, Hunt KF, Cheah YS, Forsythe E, Hazlehurst JM, Sparks K, Mohammed S, Tomlinson JW, Amiel SA, Carroll PV, Beales PL, Huda MSB, McGowan BM (2018) The endocrine and metabolic characteristics of a large Bardet-Biedl syndrome clinic population. J Clin Endocrinol Metab 103:1834-1841.

Mukhopadhyay S, Wen X, Ratti N, Loktev A, Rangell L, Scales SJ, Jackson PK (2013) The ciliary G-protein-coupled receptor Gpr161 negatively regulates the Sonic hedgehog pathway via cAMP signaling. Cell 152:210-223.

Nüsslein-Volhard C, Wieschaus E (1980) Mutations affecting segment number and polarity in Drosophila. Nature 287:795-801.

Palm W, Swierczynska MM, Kumari V, Ehrhart-Bornstein M, Bornstein SR, Eaton S (2013) Secretion and signaling activities of lipoprotein-associated hedgehog and non-sterol-modified hedgehog in flies and mammals. PLoS Biol 11:e1001505.

Pospisilik JA, Schramek D, Schnidar H, Cronin SJF, Nehme NT, Zhang X, Knauf C, Cani PD, Aumayr K, Todoric J, Bayer M, Haschemi A, Puviindran V, Tar K, Orthofer M, Neely GG, Dietzl G, Manoukian A, Funovics M, Prager G, et al (2010) Drosophila genome-wide obesity screen reveals hedgehog as a determinant of brown versus white adipose cell fate. Cell 140:148-160.

Reiter JF, Leroux MR (2017) Genes and molecular pathways underpinning ciliopathies. Nat Rev Mol Cell Biol 18:533-547.

Rodenfels J, Lavrynenko O, Ayciriex S, Sampaio JL, Carvalho M, Shevchenko A, Eaton S (2014) Production of systemically circulating Hedgehog by the intestine couples nutrition to growth and development. Genes Dev 28:2636-2651.

Rohatgi R, Milenkovic L, Scott MP (2007) Patched1 regulates hedgehog signaling at the primary cilium. Science 317:372-376.

Rouabhi M, Guo DF, Morgan DA, Zhu Z, López M, Zingman L, Grobe JL, Rahmouni K (2021) BBSome ablation in SF1 neurons causes obesity without comorbidities. Mol Metab 48:101211.

Siljee JE, Wang Y, Bernard AA, Ersoy BA, Zhang S, Marley A, Von Zastrow M, Reiter JF, Vaisse C (2018) Subcellular localization of MC4R with ADCY3 at neuronal primary cilia underlies a common pathway for genetic predisposition to obesity. Nat Genet 50:180185.

Stottmann RW, Tran PV, Turbe-Doan A, Beier DR (2009) Ttc21b is required to restrict sonic hedgehog activity in the developing mouse forebrain. Dev Biol 335:166-178.

Suh JM, Gao X, McKay J, McKay R, Salo Z, Graff JM (2006) Hedgehog signaling plays a conserved role in inhibiting fat formation. Cell Metab 3:25-34.

Sun JS, Yang DJ, Kinyua AW, Yoon SG, Seong JK, Kim J, Moon SJ, Shin DM, Choi YH, Kim KW (2021) Ventromedial hypothalamic primary cilia control energy and skeletal homeostasis. J Clin Invest 131:e138107.

Surwit RS, Kuhn CM, Cochrane C, McCubbin JA, Feinglos MN (1988) Diet-induced type II diabetes in C57BL/6J mice. Diabetes 37:1163-1167.

Traiffort E, Charytoniuk DA, Faure H, Ruat M (1998) Regional distribution of Sonic Hedgehog, patched, and smoothened mRNA in the adult rat brain. J Neurochem 70:1327-1330.

Traiffort E, Charytoniuk D, Watroba L, Faure H, Sales N, Ruat M (1999) Discrete localizations of hedgehog signalling components in the developing and adult rat nervous system. Eur $\mathrm{J}$ Neurosci 11:3199-3214.

Traiffort E, Moya KL, Faure H, Hässig R, Ruat M (2001) High expression and anterograde axonal transport of aminoterminal sonic hedgehog in the adult hamster brain. Eur J Neurosci 14:839-850.

Ugbode CI, Smith I, Whalley BJ, Hirst WD, Rattray M (2017) Sonic hedgehog signalling mediates astrocyte crosstalk with neurons to confer neuroprotection. J Neurochem 142:429-443. 
Umehara F, Tate G, Itoh K, Yamaguchi N, Douchi T, Mitsuya T, Osame M (2000) A novel mutation of desert hedgehog in a patient with $46, X Y$ partial gonadal dysgenesis accompanied by minifascicular neuropathy. Am J Hum Genet 67:1302-1305.

Vaillant C, Monard D (2009) SHH pathway and cerebellar development. Cerebellum 8:291-301.

Wang L, De Solis AJ, Goffer Y, Birkenbach KE, Engle SE, Tanis R, Levenson JM, Li X, Rausch R, Purohit M, Lee J-Y, Tan J, De Rosa
MC, Doege CA, Aaron HL, Martins GJ, Brüning JC, Egli D, Costa $\mathrm{R}$, Berbari N, et al. (2019) Ciliary gene RPGRIP1L is required for hypothalamic arcuate neuron development. JCI Insight 4:

Willaredt MA, Hasenpusch-Theil K, Gardner HAR, Kitanovic I, Hirschfeld-Warneken VC, Gojak CP, Gorgas K, Bradford CL, Spatz J, Wölfl S, Theil T, Tucker KL (2008) A crucial role for primary cilia in cortical morphogenesis. J Neurosci 28:1288712900. 